\title{
Liver Transplantation for Colorectal and Neuroendocrine Liver Metastases and Hepatoblastoma. Working Group Report From the ILTS Transplant Oncology Consensus Conference
}

\author{
Taizo Hibi, Mohamed Rela, James D. Eason, Pål-Dag Line, John Fung, Seisuke Sakamoto, Nazia
} Selzner, Kwan Man, R. Mark Ghobrial, and Gonzalo Sapisochin

\begin{abstract}
.
Liver transplantation (LT) for unresectable colorectal liver metastases has long been abandoned because of dismal prognoses. After the dark ages, advances in chemotherapy and diagnostic imaging have enabled strict patient selection, and the pioneering study from the Oslo group has contributed to the substantial progress in this field. For unresectable neuroendocrine liver metastases, LT for patients who met the Milan criteria was able to achieve excellent long-term out-comes. The guidelines further adopted in the United States and Europe were based on these criteria. For hepatoblastoma, patients with unresectable and borderline-resectable disease are considered good candidates for LT; however, the indications are yet to be defined. In the budding era of transplant oncology, it is critically important to recognize the current status and unsolved questions for each disease entity. These guidelines were developed to serve as a beacon of light for optimal patient selection for $L T$ and set the stage for future basic and clinical studies.
\end{abstract}

\section{INTRODUCTION}

Indications of liver transplantation (LT) for unresectable colorectal liver metastases (CRLM), neuroendocrine liver metastases (NETLM), and hepatoblastoma have been care- fully expanded over time. In the era of transplant oncology, LT is no more a radical approach; it is expected to play a pivotal role in multidisciplinary cancer treatment. We should be aware that there are common and diseasespecific pitfalls among these entities. The following recommendations are intended for use only by experts in specialized centers and should be interpreted carefully according to each patient's condition and the healthcare system.

\section{COLORECTAL LIVER METASTASES}

LT was considered to be contraindicated for unresectable CRLM because of the poor prognoses of $<20 \%$ survival at 5 years for patients treated in the 1980s and 1990s.1-3 The groundbreaking SECA-I (Secondary CAncer) trial was launched in 2006 at the Oslo University Hospital. The study group could allocate deceased donors to patients with CRLM because of the peculiar situation in Norway where the number of donors surpassed that of potential recipi- ents. In 2013, the investigators of SECA-I demonstrated an acceptable 5-year overall survival of $60 \%$ among 21 patients with liver-only CRLM who underwent $L T$ after at least 6 weeks of neoadjuvant chemotherapy. Four clinical factors were associated with significant worse survival: tumor diameter $>5.5 \mathrm{~cm}$, carcinoembryonic antigen $>80$ $\mu \mathrm{g} / \mathrm{L}$, time interval from resection of the primary to $\mathrm{LT}<2$ years, and progression of the metastatic disease while on chemotherapy. They were integrated to establish the Oslo score (number of risk factors ranging from 0 to 4 ) that stratifi the patients into 3 subgroups $(0-1,2-3$, and 4$)$. There were no 5 -year survivors in the subgroups of patients with all 4 factors. In addition, although 19 of 21 recipients experienced disease recurrence, the authors found 2 distinct patterns of recurrence: lungonly metastases versus multiple sites including the liver graft. It is noteworthy that the type of recurrence signify impacted survival.5 Furthermore, the SECA-II trial investigated colorectal cancer patients who underwent LT for nonresectable liver-only metastases confi by computed tomography/magnetic resonance imaging/positron emission tomography, with at least $10 \%$ response to chemotherapy, and with a time interval between diagnosis to LT of $>1$ year. Under these more stringent selection criteria compared with those of SECA-I, the study group reported even better outcomes of $83 \%$ overall sur- 
vival at 5 years for 15 patients. 6 More recently, the same group described that 13 of 19 patients (14 and 5 patients from the SECA-I and SECA-II trials, respectively) with an Oslo score of 0-2 enjoyed a 5year overall survival of $67 \%$ compared with $17 \%$ in patients with an Oslo score of $3-4$, with a median follow-up of 85 months. 7 The authors under- scored that disease-free survival should not be used as an outcome measure to assess the effi of LT for CRLM because recurrence alone is not predictive of survival.7 It is noteworthy that all patients in the SECA-I and SECA-II trials received sirolimus (a mammalian target of rapamycin inhibitor) for maintenance immunosuppression. Meanwhile, a report from the Compagnons Hepato-Biliaires demonstrated that 5 of 12 recipients were alive without evidence of recurrence at 7 to 108 months after LT.8 This study revealed that, in contrast to the Norwegian experience, long-term disease-free survival can be achieved in carefully selected patients who had previously undergone a combination of multiple courses of chemotherapy and liver resections. To build on the momentum, multiple European and US cent- ers have launched prospective trials of $\mathrm{LT}$ for CRLM. Total 7 prospective studies including randomized control trials have been registered at ClinicalTrials.gov at the time of this report (NCT01479608, NCT02215889, NCT02597348, NCT02864485, NCT03494946, NCT03488953, and NCT03803436). There is another ongoing trial called the Porto Alegre protocol of the Compagnons Hepato-Biliaires. 3 It is obvious that the recent success of LT for unresectable CRLM is due to highly effective chemotherapy, advanced imaging technology (eg, positron emission tomography to rule out extrahepatic disease), 9 better understanding of tumor biology (eg, BRAF mutation), 10 improved perioperative management of LT, etc. Currently, the most essential clinical question is about patient selection. Although the Oslo group has proposed a scoring system to predict favorable prognoses at a population level, the results of other ongoing trials are awaited to defi the ideal candidate for LT for CRLM. Other unsolved questions include the following: (1) acceptable outcomes after LT in the context of persistent organ shortage at a community level7; (2) how to incorporate LT in the multidisciplinary care of CRLM at an individual level, that is, defi of "unresectable" remains ill-defi 11,12 and the possibility of LT for "resect- able" disease, 13 the use of adjuvant chemotherapy after LT, and the role of mammalian target of rapamycin inhibitors versus calcineurin inhibitors to optimize immunosuppression after LT should also be discussed; and (3) how to meet the potentially high demand in an era of colorectal cancer epidemic (ie, RAPID concept).13-15

\section{Recommendations}

1. LT can be a viable option in highly selected patients with unresectable CRLM with only liver involvement (moderate level of evidence and moderate recommendation).

2. LT for CRLM with low Oslo score $\leq 2$ (maximum tumor diameter $\leq 5.5 \mathrm{~cm}$, pretransplant carcinoembryonic anti- gen $\leq 80 \mu \mathrm{g} / \mathrm{L}$, response to chemotherapy, time interval: diagnosis to $\mathrm{LT} \geq 2 \mathrm{y}$ ) may improve the 5-year overall survival rates over those achieved with the current stand-ard of care (moderate level of evidence and moderate recommendation).

3. Minimization of immunosuppression is recommended (low level of evidence and moderate recommendation).

4. Aggressive treatment of all posttransplant resectable recurrences is recommended (low level of evidence and moderate recommendation).

5. There is a need for an international registry to coordinate data collection and design further studies on LT for CRLM (moderate level of evidence and moderate recommendation).

\section{NEUROENDOCRINE LIVER METASTASES}

The largest systematic review on LT for NETLM that has been conducted till date has studied $>1100$ patients based on registries and multicenter and single-center stud- ies.16 Multivisceral transplantation was applied for cases of widespread disease and involvement of other organs for $<20 \%$ of the entire cohort. The reported 5 -year overall survival rate was $63 \%$ after LT for unresectable NETLM, with a recurrence rate raging between $30 \%$ and $60 \%$. More than $50 \%$ liver involvement, a high Ki67 index, and pancreatic NET versus gastrointestinal NET as the primary lesion 
were predictive factors of decreased long-term survival.16 In 2007, Mazzaferro et al17 stated that LT for NETLM should be conducted with the intent of cure rather than palliation, based on previous reports that liver resection commonly does not provide sufficient out- comes for patients with severe symptoms caused by dif- fuse disease and/or carcinoid syndrome. They developed the Milan criteria for NETLM: confirmed histology of G1 or G2 tumor (World Health Organization Classification of Neuroendocrine Tumors 2010), the primary tumor drained by the portal system, hepatic involvement of $<50 \%$, com- plete resection of primary tumor and all extrahepatic dis- ease with stable disease or good response to therapies for at least 6 months, and age $<60$ years (relative criteria). In their prospective study, remarkable 5 - and 10 -year survival rates of $97 \%$ and $89 \%$, respectively, were achieved in 42 highly selected patients who underwent LT between 1995 and 2010.18 These numbers were significantly better than those of $51 \%$ and $22 \%$, respectively, in the 46 patients with similar tumor burden who were treated with a non- transplant strategy. In the LT group, the time-toprogression at 10 years was only $13 \%$, a percentage that has never been described in patients with unresectable NETLM. In the United States, United Network for Organ Sharing has adopted the Milan criteria and released guidelines to list the potential candidates with unresectable NETLM for LT.19 Other existing guidelines include the European Neuroendocrine Tumor Society Consensus Guidelines and the National Comprehensive Cancer Network Guidelines; however, universal criteria have yet to be established.20,21 Other important questions include the following: (1) how to incorporate nonoperative modalities that have changed the paradigm of treatment for NET (eg, peptide receptor radionuclide therapy and molecular-targeted agents)22,23 as downstaging/bridge therapy before LT24; (2) standardization of imaging protocol to accurately diagnose pre- LT patients and detect postLT recurrence25,26; (3) use of everolimus post-LT for both immunosuppression 27 and adjuvant therapy; (4) what are the appropriate outcome indicators (overall survival versus time to progression, etc) given the indolent clinical course of NET; (5) definition of unresectability or if there is any room to adopt LT for resectable, bulky disease1; and (6) whether LT would provide survival benefit for patients with more aggressive disease given their low response rate to conventional therapies.13

\section{Recommendations}

1. LT should be considered as a potentially curable treatment option for selected patients with unresectable metastatic NET of midgut/hindgut origin confined to the liver (moderate level of evidence and strong recommendation).

2. Selection criteria should consider 68Ga-DOTATATE, Ki67, histology, site of origin, and a certain time interval of stable disease or good response to therapies (moderate level of evidence and strong recommendation).

3. LT for selected patients with metastatic NET confined to the liver as part of multimodality therapy should achieve comparable outcomes as LT for other diagnoses (moderate level of evidence and strong recommendation).

4. Everolimus has achieved improvement in progression-free survival in NET and should be considered as part of immunosuppression after LT for NETLM (low level of evidence and strong recommendation).

5. Late recurrences beyond 5 years after $L T$ are not uncommon, necessitating long-term followup with annual imaging (moderate level of evidence and strong recommendation).

\section{HEPATOBLASTOMA}

Hepatoblastoma is the most common primary liver cancer in children and its incidence has continued to increase over the past 2 decades. Surgical resection with chemotherapy is the mainstay of treatment.28-30 The degree of tumor burden and prognoses are determined as per the pretreatment and posttreatment extent of disease (PRETEXT and POST-TEXT) system.31,32 Signifi advances in chemo- therapy33-35 and perioperative management have yielded an overall 5-year survival of $60 \%$ to $>80 \%$ after LT for unresectable hepatoblastoma and is considered a standard treatment worldwide.36-40 The current Children's Oncology Group international study of pediatric liver cancer 
AHEP- 1531 adopted a novel risk stratify algorithm established by the Children's Hepatic tumors International

Collaboration.32,40 Patients with PRETEXT III hepatoblastomas that are deemed unresectable after the 2 cycles of chemotherapy cycle, PRETEXT IV hepatoblastomas, and

POST-TEXT III and IV hepatoblastomas should receive early referral to a specialized center with extensive experience in LT and complex liver resection.41-43 Even patients with extrahepatic disease are eligible for LT, provided the lesions have disappeared with chemotherapy or been surgically removed.37,44,45 LT should also be considered in borderline-resectable hepatoblastoma because the outcomes after salvage transplantation are unsatisfactory.46,47 A recent study demonstrated that there was no significant difference in 10-year patient survival after LT between patients with unresectable malignant primary pediatric hepatic tumors (81\%) and those with nonmalignant causes (88\%).48 Indocyanine green (ICG) has been demonstrated to be use- ful in the detection of metastatic deposits.49,50 Living donors are an indispensable source of liver grafts and can optimize the timing of LT.38,51 Correspondingly, patients with hepatoblastoma on chemotherapy should be prioritized on the waiting list in deceased donor LT.52 Although LT is widely accepted as a lifesaving option for unresectable hepatoblastoma, its universal indication is yet to be defi 29 The SIOPEL (Société Internationale d'Oncologie Pédiatrique- Epithelial Liver) group has published guidelines and listed the following conditions to strongly encourage early referral to LT programs: multifocal PRETEXT IV hepatoblastoma; large solitary PRETEXT IV hepatoblastoma unresponsive to preoperative chemotherapy; unifocal, centrally located tumors involving main hilar structures or main hepatic veins that is unlikely to become tumor free even after good

response to chemotherapy.53 In a single-center analysis, longer time on the waiting list was identifi as a risk factor of recurrence after LT.52 The early referral system is criti- cal to shorten the interval between diagnosis and LT54 and reduce the risk of recurrence, which is a strong predictive factor of diminished survival.39 Further genetic studies and molecular analyses are warranted to realize precision medi- cine for hepatoblastoma.55,56

\section{Recommendations}

1. Hepatoblastoma has excellent outcomes with a multidisciplinary approach. Surgery with chemotherapy has resulted in 5-year overall survival of up to $80 \%$ (high level of evidence and strong recommendation). Cisplatin-based CT has improved resectability rates from $30 \%$ to $75 \%-80 \%$ (moderate level of evidence and strong recommendation).

2. PRETEXT and POST-TEXT staging system with cross- sectional imaging is useful for risk stratification and treatment. High-risk factors include PRETEXT IV, age $>3$ years, extrahepatic metastases, alpha-fetoprotein level $<100 \mathrm{ng} / \mathrm{mL}$, and major bilobar vascular involvement (moderate level of evidence and strong recommendation).

3. The risk stratified treatment protocol has helped in achieving excellent outcomes with minimal chemotoxicity. Six cycles of cisplatin monotherapy (adjuvant \pm neoadjuvant) are recommended for non-well-differentiated fetal histology tumors (moderate level of evidence and strong recommendation).

4. Surgical resection is the mainstay of therapy in low-risk tumors (moderate level of evidence and strong recommendation).

5. Patients with high-risk tumors or those requiring complex liver surgery or transplantation should be referred early to specialized centers (low level of evidence and strong recommendation).

6. LT has increased resectability by $25 \%-30 \%$ in the high- risk group and has achieved long-term survival rates $>80 \%$. Unifocal POST-TEXT IV tumors and/or POST-TEXT III or IV with persistent widespread multifocality or major vessel involvement are clear indications for LT (moderate level of evidence and strong recommendation).

7. LT is indicated for patients with treatment-responsive metastatic disease (moderate level of evidence and strong recommendation). ICG may be helpful in identifying viable tumors to guide surgical therapy (low level of evidence and moderate recommendation). 
8. Living donor LT can optimize the timing of surgery between chemotherapy sessions (low level of evidence and strong recommendation). Patients with hepatoblastoma on chemotherapy should be prioritized for deceased donor allocation to optimize the timing of LT (low level of evidence and moderate recommendation).

9. Studies on tumor genetic and molecular analysis can help in better prognostication and chemo responsiveness (low level of evidence and moderate recommendation).

\section{REFERENCES}

1. Hibi T, Sapisochin G. What is transplant oncology? Surgery. 2019;165:281-285.

2. Gorgen A, Muaddi $\mathrm{H}$, Zhang W, et al. The new era of transplant oncology: liver transplantation for nonresectable colorectal cancer liver metastases. Can J Gastroenterol Hepatol. 2018;2018:9531925.

3. Andres A, Oldani G, Berney $\mathrm{T}$, et al. Transplantation for colorectal metastases: on the edge of a revolution. Transl Gastroenterol Hepatol. 2018;3:74.

4. Hagness $M$, Foss $A$, Line PD, et al. Liver transplantation for non- resectable liver metastases from colorectal cancer. Ann Surg. 2013;257:800-806.

5. Hagness $M$, Foss A, Egge TS, et al. Patterns of recurrence after liver transplantation for nonresectable liver metastases from colorectal cancer. Ann Surg Oncol. 2014;21:1323-1329.

6. Dueland S, Syversveen T, Solheim JM, et al. Survival following liver transplantation for patients with nonresectable liver-only colorectal metastases. Ann Surg. 2020;271:212-218.

7. Dueland S, Grut H, Syversveen T, et al. Selection criteria related to long term survival following liver transplantation for colorectal liver metastasis. Am J Transplant. 2020;20:530-537.

8. Toso C, Pinto Marques H, Andres A, et al; Compagnons Hépato- Biliaires Group. Liver transplantation for colorectal liver metasta- sis: survival without recurrence can be achieved. Liver Transpl. 2017;23:1073-1076.

9. Grut H, Revheim ME, Line PD, et al. Importance of 18F-FDG PET/CT to select patients with nonresectable colorectal liver metastases for liver transplantation. Nucl Med Commun. 2018;39:621627.

10. Tol J, Nagtegaal ID, Punt CJ. BRAF mutation in metastatic colorectal cancer. N Engl J Med. 2009;361:98-99.

11. Huiskens J, Bolhuis K, Engelbrecht MR, et al; Dutch Colorectal Cancer Group. Outcomes of resectability assessment of the Dutch Colorectal Cancer Group liver metastases expert panel. J Am Coll Surg. 2019;229:523-532.e2.

12. Imai K, Adam R, Baba H. How to increase the resectability of initially unresectable colorectal liver metastases: a surgical perspective. Ann Gastroenterol Surg. 2019;3:476-486.

13. Abreu P, Gorgen A, Oldani G, et al. Recent advances in liver trans- plantation for cancer: the future of transplant oncology. JHEP Rep. 2019;1:377-391.

14. Line $P D$, Hagness $M$, Berstad $A E$, et al. A novel concept for partial liver transplantation in nonresectable colorectal liver metastases: the RAPID concept. Ann Surg. 2015;262:e5-e9.

15. Königsrainer A, Templin S, Capobianco I, et al. Paradigm shift in the management of irresectable colorectal liver metastases: living donor auxiliary partial orthotopic liver transplantation in combination with two-stage hepatectomy (LD-RAPID). Ann Surg. 2019;270:327-332.

16. Moris D, Tsilimigras DI, Ntanasis-Stathopoulos I, et al. Liver transplan- tation in patients with liver metastases from neuroendocrine tumors: a systematic review. Surgery. 2017;162:525-536.

17. Mazzaferro V, Pulvirenti A, Coppa J. Neuroendocrine tumors meta- static to the liver: how to select patients for liver transplantation? J Hepatol. 2007;47:460-466.

18. Mazzaferro V, Sposito C, Coppa J, et al. The long-term benefit of liver transplantation for hepatic metastases from neuroendocrine tumors. Am J Transplant. 2016;16:2892-2902.

19. US Department of Health and Human Services. Guidance to Liver Transplant Programs and the National Liver Review Board for: Adult 
MELD Exception Review. US Department of Health and Human Services website. Available at https://optnpilot.unos.org/media/2847/liver_guidance_adult_meld_201706.pdf. Accessed October 30, 2019.

20. National Cancer Comprehensive Network. Neuroendocrine and adrenal tumors (Version 1.2019). National Cancer Comprehensive Network website. Available at https://www.nccn.org/professionals/ physician_gls/pdf/neuroendocrine.pdf. Accessed October 30, 2019.

21. Pavel M, O'Toole D, Costa F, et al; Vienna Consensus Conference participants. ENETS consensus guidelines update for the manage- ment of distant metastatic disease of intestinal, pancreatic, bronchial neuroendocrine neoplasms (NEN) and NEN of unknown primary site. Neuroendocrinology. 2016;103:172-185.

22. Strosberg J, El-Haddad G, Wolin E, et al; NETTER-1 Trial Investigators. Phase 3 trial of 177ludotatate for midgut neuroendocrine tumors. N EngI J Med. 2017;376:125-135.

23. Yao JC, Fazio N, Singh S, et al; RAD001 in Advanced Neuroendocrine Tumours, Fourth Trial (RADIANT-4) Study Group. Everolimus for the treatment of advanced, non-functional neuroendocrine tumours of the lung or gastrointestinal tract (RADIANT-4): a randomised, placebo- controlled, phase 3 study. Lancet. 2016;387:968-977.

24. Frilling A, Clift AK. Therapeutic strategies for neuroendocrine liver metastases. Cancer. 2015;121:1172-1186.

25. Bodei L, Ambrosini V, Herrmann K, et al. Current concepts in 68ga- DOTATATE imaging of neuroendocrine neoplasms: interpretation, biodistribution, dosimetry, and molecular strategies. $J$ Nucl Med. 2017;58:1718-1726.

26. Chan DL, Pavlakis N, Schembri GP, et al. Dual somatostatin recep- tor/FDG PET/CT imaging in metastatic neuroendocrine tumours: proposal for a novel grading scheme with prognostic significance. Theranostics. 2017;7:1149-1158.

27. De Simone P, Fagiuoli S, Cescon M, et al; Consensus Panel. Use of everolimus in liver transplantation: recommendations from a working group. Transplantation. 2017;101:239-251.

28. Meyers RL, Tiao GM, Dunn SP, et al; Central Surgical Review Committee, Children's Oncology Group AHEP-0731, Treatment of Children with All Stages Hepatoblastoma. Surgical manage- ment of children with locally advanced hepatoblastoma. Cancer. 2012;118:4090-4091. Author reply 4094.

29. Zsíros J, Maibach R, Shafford E, et al. Successful treatment of child- hood high-risk hepatoblastoma with dose-intensive multiagent chem- otherapy and surgery: final results of the SIOPEL-3HR study. J Clin Oncol. 2010;28:2584-2590.

30. Ortega JA, Douglass EC, Feusner JH, et al. Randomized comparison of cisplatin/vincristine/fluorouracil and cisplatin/continuous infusion doxorubicin for treatment of pediatric hepatoblastoma: a report from the Children's Cancer Group and the Pediatric Oncology Group. J Clin Oncol. 2000;18:2665-2675.

31. Towbin AJ, Meyers RL, Woodley H, et al. 2017 PRETEXT: radiologic staging system for primary hepatic malignancies of childhood revised for the Paediatric Hepatic International Tumour Trial (PHITT). Pediatr Radiol. 2018;48:536-554.

32. Meyers RL, Maibach R, Hiyama E, et al. Risk-stratified staging in pae- diatric hepatoblastoma: a unified analysis from the children's hepatic tumors international collaboration. Lancet Oncol. 2017;18:122-131.

33. Czauderna P, Lopez-Terrada D, Hiyama E, et al. Hepatoblastoma state of the art: pathology, genetics, risk stratification, and chemo- therapy. Curr Opin Pediatr. 2014;26:19-28.

34. Czauderna P, Haeberle B, Hiyama E, et al. The children's hepatic tumors international collaboration (CHIC): novel global rare tumor database yields new prognostic factors in hepatoblastoma and becomes a research model. Eur J Cancer. 2016;52:92-101.

35. Watanabe K. Current chemotherapeutic approaches for hepatoblas- toma. Int J Clin Oncol. 2013;18:955-961. 
36. Faraj W, Dar F, Marangoni G, et al. Liver transplantation for hepato- blastoma. Liver Transpl. 2008;14:1614-1619.

37. Cruz RJ Jr, Ranganathan S, Mazariegos G, et al. Analysis of national and single-center incidence and survival after liver transplantation for hepatoblastoma: new trends and future opportunities. Surgery. 2013;153:150-159.

38. Sakamoto S, Kasahara M, Mizuta K, et al; Japanese Liver Transplantation Society. Nationwide survey of the outcomes of living donor liver transplantation for hepatoblastoma in Japan. Liver Transpl. 2014;20:333-346.

39. Ramos-Gonzalez G, LaQuaglia M, O'Neill AF, et al. Long-term out- comes of liver transplantation for hepatoblastoma: a single-center 14-year experience. Pediatr Transpl. 2018:e13250.

40. Lim IIP, Bondoc AJ, Geller JI, et al. Hepatoblastoma-the evolution of biology, surgery, and transplantation. Children (Basel). 2018;6:1.

41. Lautz TB, Ben-Ami T, Tantemsapya N, et al. Successful nontrans- plant resection of POST-TEXT III and IV hepatoblastoma. Cancer. 2011;117:1976-1983.

42. Fuchs J, Cavdar S, Blumenstock G, et al. POST-TEXT III and IV hepa- toblastoma: extended hepatic resection avoids liver transplantation in selected cases. Ann Surg. 2017;266:318-323.

43. Guérin F, Gauthier F, Martelli H, et al. Outcome of central hepatectomy for hepatoblastomas. J Pediatr Surg. 2010;45:555-563.

44. Perilongo G, Brown J, Shafford E, et al. Hepatoblastoma presenting with lung metastases: treatment results of the first cooperative, pro- spective study of the International Society of Paediatric Oncology on childhood liver tumors. Cancer. 2000;89:1845-1853.

45. O'Neill AF, Towbin AJ, Krailo MD, et al. Characterization of pulmonary metastases in children with hepatoblastoma treated on Children's Oncology Group Protocol AHEP0731 (the treatment of children with all stages of hepatoblastoma): a report from the Children's Oncology Group. J Clin Oncol. 2017;35:3465-3473.

46. Otte JB, Pritchard J, Aronson DC, et al; International Society of Pediatric Oncology (SIOP). Liver transplantation for hepatoblastoma: results from the International Society of Pediatric Oncology SIOP) study SIOPEL-1 and review of the world experience. Pediatr Blood Cancer. 2004;42:74-83.

47. Héry $G$, Franchi-Abella $S$, Habes $D$, et al. Initial liver transplantation for unresectable hepatoblastoma after chemotherapy. Pediatr Blood Cancer. 2011;57:1270-1275.

48. Khan AS, Brecklin B, Vachharajani $\mathrm{N}$, et al. Liver transplantation for malignant primary pediatric hepatic tumors. J Am Coll Surg. 2017;225:103-113.

49. Kitagawa N, Shinkai M, Mochizuki K, et al. Navigation using indo- cyanine green fluorescence imaging for hepatoblastoma pulmonary metastases surgery. Pediatr Surg Int. 2015;31:407-411.

50. Takahashi N, Yamada Y, Hoshino K, et al. Living donor liver re-trans- plantation for recurrent hepatoblastoma in the liver graft following complete eradication of peritoneal metastases under indocyanine green fluorescence imaging. Cancers (Basel). 2019;11:730.

51. Uchida H, Sakamoto S, Sasaki K, et al. Surgical treatment strategy for advanced hepatoblastoma: resection versus transplantation. Pediatr Blood Cancer. 2018;65:e27383.

52. Pham TA, Gallo AM, Concepcion W, et al. Effect of liver transplant on long-term disease-free survival in children with hepatoblastoma and hepatocellular cancer. JAMA Surg. 2015;150:11501158.

53. Czauderna P, Otte JB, Aronson DC, et al; Childhood Liver Tumour Strategy Group of the International Society of Paediatric Oncology (SIOPEL). Guidelines for surgical treatment of hepatoblastoma in the modern era-recommendations from the Childhood Liver Tumour Strategy Group of the International Society of Paediatric Oncology (SIOPEL). Eur J Cancer. 2005;41:1031-1036.

54. Kueht $M$, Thompson $P$, Rana $A$, et al. Effects of an early referral sys- tem on liver transplantation for hepatoblastoma at Texas Children's Hospital. Pediatr Transplant. 2016;20:515522. 
55. Ueda Y, Hiyama E, Kamimatsuse A, et al. Wnt signaling and telomerase activation of hepatoblastoma: correlation with che- mosensitivity and surgical resectability. J Pediatr Surg. 2011;46: 2221-2227.

56. Takayasu $\mathrm{H}$, Horie $\mathrm{H}$, Hiyama $\mathrm{E}$, et al. Frequent deletions and muta- tions of the beta-catenin gene are associated with overexpression of cyclin D1 and fibronectin and poorly differentiated histology in child- hood hepatoblastoma. Clin Cancer Res. 2001;7:901-908. 